\title{
Thermally Stimulated Depolarization Currents (TSDC): A sensitive technique for analyzing protein structure
}

\author{
R. Bedotti, M.G. Bridelli *, R. Capelletti and E. Polverini \\ Department of Physics and CNISM, University of Parma, Parma, Italy
}

\begin{abstract}
The water molecules surrounding proteins as a thin layer and those packaged in pockets and cavities shape and control their structure. Thermally Stimulated Depolarization Currents (TSDC) technique has been applied to investigate the hydration structure of six proteins with different structural motifs: pepsin, $\beta$-lactoglobulin, $\alpha$-chymotrypsin, bovine serum albumin, human serum albumin and myoglobin, at very low hydration level (water vapor activity $a_{\mathrm{w}} \approx 0.80$ ) both in the native state and after treatment in trifluoroethanol/water mixture $80 \%(\mathrm{v} / \mathrm{v})$. A combined approach based on the use of the TSDC technique, able to distinguish $\mathrm{H}_{2} \mathrm{O}$ dipoles belonging to the solvation shell in terms of their order degree and mobility, and of FTIR and CD spectroscopies has allowed us to reexamine the problem of conformational stability of macromolecules as a function of their hydration.
\end{abstract}

Keywords: Thermally Stimulated Depolarization Currents (TSDC), FTIR, CD, hydration, protein structure, TFE

\section{Introduction}

It is well known that proteins exist and act in a predominantly aqueous solvent environment. In fact, water controls and affects many features of structure and function of biological macromolecules. The accepted model of protein hydration assumes the existence of discrete water-binding sites both within clefts and cavities and on the polypeptide surface. TSDC is a dielectric technique which was proved to be a powerful tool for studying hydration water in biological macromolecules being able to distinguish water dipoles with different mobility in the temperature domain. It is based on the water microdynamics which depends on the surrounding: as a consequence, families of water can be distinguished by different dielectric relaxation times and different statistical weights. The method is characterized by high sensitivity and resolving power: dipole concentration as low as $0.1 \mathrm{ppm}$ can be accurately measured. The typical TSDC spectrum (a plot of the depolarization current as a function of the temperature) for a protein consists, as a rule, of two main peaks, generally called Low Temperature (LT) and High Temperature (HT) peak, respectively, the former being related to water molecules located in the grooves of the folded structure and the latter to the external ones, layered on the polypeptide surface. A recent TSDC study, performed on poly-L-lysine in different conformational states, has suggested that the solvent arrangement around the polypeptide structure depends almost exclusively on the protein surface

\footnotetext{
${ }^{*}$ Corresponding author: M.G. Bridelli, Department of Physics, University of Parma, Viale G.P. Usberti 7/A, Parco Area delle Scienze, 43100 Parma, Italy. Tel.: +39 0521 906227; Fax: +39 0521 905223; E-mail: mariagrazia.bridelli@ unipr.it.
} 
geometry and on the hydrogen bonding capability [1]. On these bases, TSDC measurements were carried out on lysozyme to explore the conformational changes accompanying the transition of the protein into the amyloid form [1]. The results showed large differences between the TSDC spectra related to the two different protein conformations, for what concerns the number and position of the main peaks. In fact, the native form displayed two peaks, at $T_{\mathrm{M}}=175 \mathrm{~K}$ and at $T_{\mathrm{M}}=297 \mathrm{~K}$, while the amyloid one exhibited only a peak at intermediate temperature $\left(T_{\mathrm{M}}=235 \mathrm{~K}\right)[1]$.

With the aim to investigate how the molecular geometry and the hydration are related by analyzing the TSDC spectra dependence on the protein conformational structure, six proteins of different conformational types were considered in the present work: pepsin, $\beta$-lactoglobulin, $\alpha$-chymotrypsin (mainly-beta proteins), bovine serum albumin (BSA), human serum albumin (HSA) and myoglobin (mainly-alpha proteins). They were studied in the native form and after treatment in 2,2,2-trifluorethanol (TFE)/water mixture $80 \%(\mathrm{v} / \mathrm{v})$. This organic solvent has been shown to promote $\alpha$-helical secondary structure formation by replacing the hydration shell with fluoroalchohol molecules, a process inducing protein dehydration [4]. The TSDC analysis of conformational transitions induced in proteins by TFE was associated with CD spectroscopy and FTIR measurements in the Amide I region.

\section{Experimental details}

All the proteins were used without further chemical purification. To modify the secondary structure the proteins were incubated with $80 \%$ (v/v) TFE at room temperature (RT) for 1 day. The same hydration degree was obtained for all the samples to submit to TSDC measurements by equilibrating them in a dry box for a week over saturated $\mathrm{NH}_{4} \mathrm{Cl}$ solution, providing a water vapor activity $a_{\mathrm{w}} \approx 0.80$.

\subsection{TSDC measurements}

Samples were pellets of $13 \mathrm{~mm}$ diameter and $0.85-1.00 \mathrm{~mm}$ thickness, obtained by applying a pressure of $7.4 \times 10^{8} \mathrm{~Pa}$ to $150 \mathrm{mg}$ of powders. They were placed in a closed vessel, where a controlled gaseous atmosphere was established to minimize dehydration of the samples. Metal electrodes with plane symmetry were employed. A standard apparatus was used [3] and the spectra were recorded in the temperature range $100-300 \mathrm{~K}$. An electric field $E_{\mathrm{p}} \approx 2 \times 10^{3} \mathrm{~V} / \mathrm{cm}$ was applied to the sample for a polarization time $t_{\mathrm{p}} \approx 3 \mathrm{~min}$. The heating rate was about $0.15 \mathrm{~K} / \mathrm{s}$. For the technique theory, see $[1,3]$.

\subsection{FTIR measurements}

FTIR measurements were performed on thin films obtained by depositing aqueous solutions $(c=$ $10 \mathrm{mg} / \mathrm{ml}$ ) on $\mathrm{CaF}_{2}$ windows and allowing them to dry in air. The spectra were collected by means of a Jasco 420 FTIR spectrophotometer operating at RT in the transmission mode (4000-600 $\mathrm{cm}^{-1}$ wave number range, 128 scans, spectral resolution of $2 \mathrm{~cm}^{-1}$ ). The amide bands were fit according to Gaussian curves by means of Origin 8.0 program.

\subsection{CD measurements}

Far UV (185-250 nm) CD spectra were recorded by using a Jasco J715 spectropolarimeter, with an optical pathway of $0.01 \mathrm{~cm}$, averaging 3 scans and collecting data every $0.5 \mathrm{~nm}$. The solvent background was always subtracted. The proteins were dissolved in water or in a mixture of water/TFE $80 \%(\mathrm{v} / \mathrm{v})$, at 

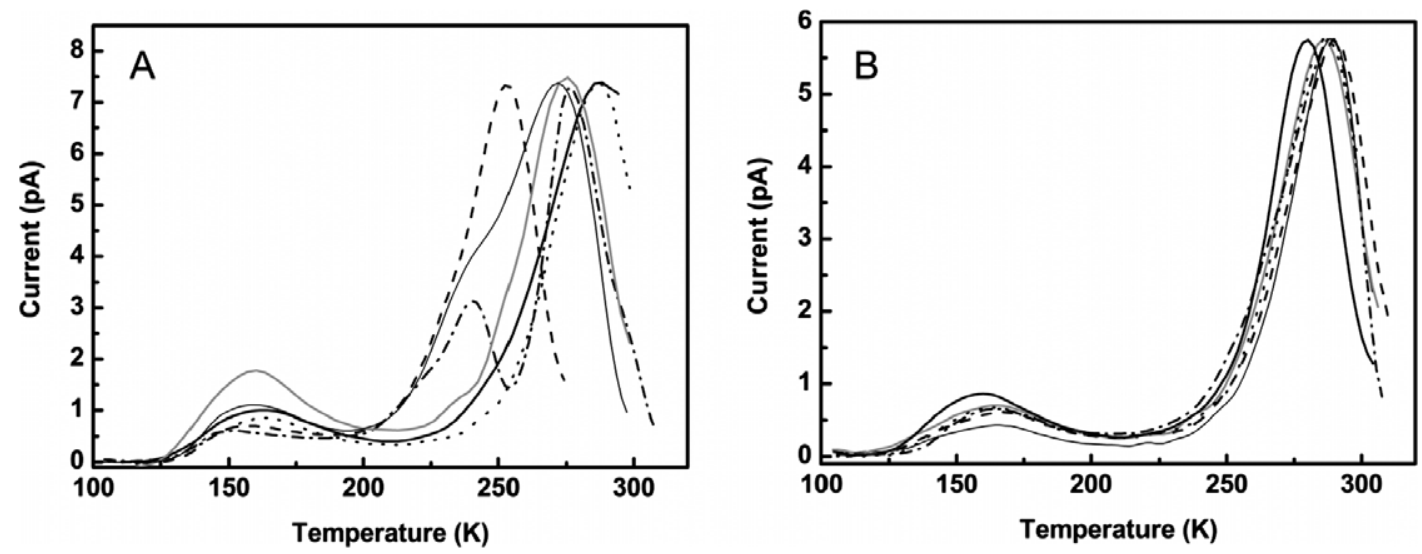

Fig. 1. TSDC spectra of six proteins in the native (A) form and after treatment with TFE ( $80 \% \mathrm{v} / \mathrm{v})(\mathrm{B})$ ( - $\alpha$-chymotrypsin, $--\beta$-lactoglobulin; $\bullet \bullet \bullet$ BSA, $-\bullet-$ HSA, $\longrightarrow$ myoglobin, - pepsin).

such a concentration as the absorbance at $278 \mathrm{~nm}$ lay between 0.05 and 0.1 . In these conditions, the concentrations of the different proteins were as follows: pepsine $2.9 \mu \mathrm{M}$; BSA $1.8 \mu \mathrm{M}$; myoglobin $5.7 \mu \mathrm{M}$; $\alpha$-chymotrypsin $1.6 \mu \mathrm{M}$; HSA $2.3 \mu \mathrm{M}$. The proteins concentration was evaluated spectrophometrically. The CD spectra were analyzed by using the algorithms embedded into the software package CDPro [5] using the SELCON3, CDSSTR and CONTINLL methods.

\section{Results and discussion}

Figure 1A shows the TSDC spectra obtained by polarizing the samples of the six proteins in a wide temperature range $\left(T_{\mathrm{p}}=300 \mathrm{~K} ; T_{\mathrm{f}}=100 \mathrm{~K}\right)$. To make the comparison easier, the spectra are normalized to the maximum height. All the spectra display two main peaks, as expected: a weak one at $T_{\mathrm{M}} \approx 170 \mathrm{~K}$ (LT peak) and a large and broad one near room temperature (HT peak). By a proper choice of the polarization range $T_{\mathrm{p}}-T_{\mathrm{f}}$ many components of the complex spectra were put in evidence, revealing that they are characterized by a wide distribution of activation energies and relaxation times. The present results and the comparison with those obtained in the past on other proteins suggested to attribute the peaks to water molecules, belonging to several classes of dipoles endowed with different mobility and coordinated by the polypeptides. On the basis of the temperature range at which the peaks appear, the LT peak can be related to water dipoles buried in the interior of the macromolecules and the HT peak to the water decorating the surfaces. It is well established, in fact, that the dynamical properties of water molecules and their residence times change as a continuum between the tightly bound $\mathrm{H}_{2} \mathrm{O}$ molecules, hosted in protein interiors, and the bulklike molecules in the external layer of the protein. The variety of shapes and positions, exhibited mainly by the HT peak of the different proteins deserves particular notes: in some proteins it appears large and broad, in others it displays several features as shoulders. These differences may originate from the reorientation of water dipoles organized around different protein surfaces, due to different secondary structure. Further insight in the structural characterization has been obtained by means of CD and FTIR spectroscopies. Figure 2A shows the CD spectra of the six proteins in the native state and Fig. 2B the detail of the FTIR spectrum related to Amide I band for one of them (HSA). The analysis of the CD spectra confirms the presence of the structural motifs, whose percentages are in accordance with the literature data. The decomposition of the Amide I feature in 

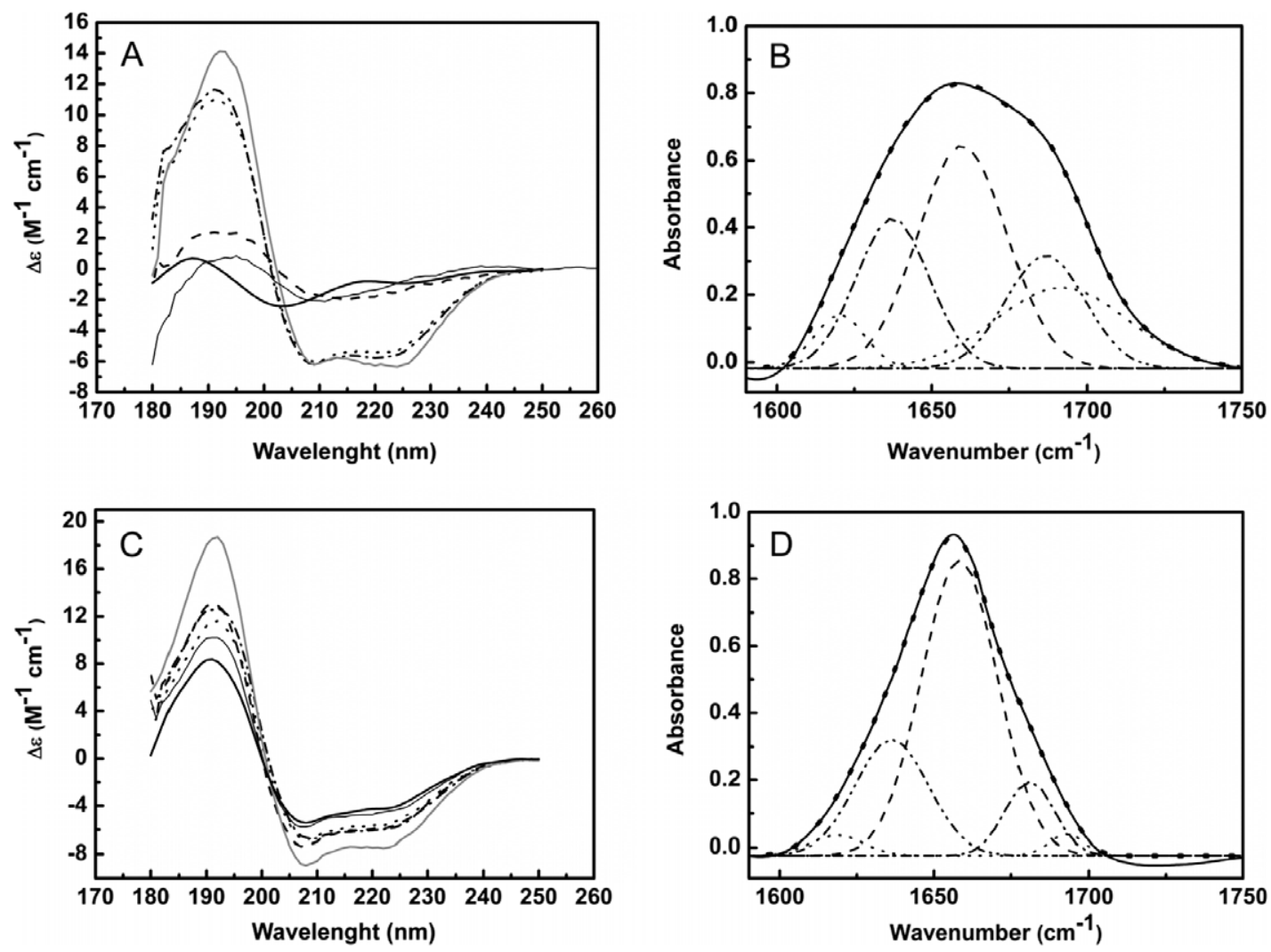

Fig. 2. Role of the addition of TFE to proteins, as monitored by means CD and FTIR spectroscopies. (A and C) CD spectra before and after treatment ( $-\alpha$-chymotrypsin, $--\beta$-lactoglobulin; $\bullet \bullet$ BSA, $-\bullet-$ HSA, - myoglobin, - pepsin). (B and D) Gaussian deconvolution of FTIR spectra in the Amide I region for HSA before and after treatment with TFE. The sum of the fitted curves is shown by the dotted line overlapping the experimental curve (full line).

Gaussian components reveals a mean peak at $\sim 1655 \mathrm{~cm}^{-1}$ which indicates the predominance of an $\alpha$-helical structure in HSA. Additional maxima suggest the presence of minor contributions due to $\beta$ and unordered structures. As a consequence of the addition of TFE to the proteins, the TSDC spectra dramatically change and, surprisingly, all the spectra of the treated proteins show similar features: LT peaks do not change meaningfully, HT peaks, on the contrary, shift towards higher temperature region, all displaying the maximum at the same temperature $\left(T_{\mathrm{M}} \approx 290 \mathrm{~K}\right)$. An exception is represented by $\alpha$-chymotrypsin, which exhibits a small shift, with negligible shape changes (Fig. 1B). The amplitudes of the peaks change as well; as for Fig. 1A, the spectra are normalized to the maximum height. It is noteworthy that the spectra of the proteins treated with TFE resemble closely those of proteins submitted to water desorption treatment, previously studied. Such a conclusion is along with the model proposed for TFE interaction with peptides, a process involving the macromolecule dehydration. Figure $2 \mathrm{C}$ and $2 \mathrm{D}$ portray the CD and FTIR spectra of the proteins treated with TFE. The comparison of the CD spectra of proteins in aqueous solution with those in water/TFE mixture shows an evident conversion of the whole protein towards an $\alpha$-helical structure. All the spectra, in fact, adopt the typical $\alpha$-helix pattern with comparable intensities. If the structural transition is expected in proteins that in water contain a large fraction of beta structure (chymotrypsin, $\beta$-lactoglobulin, pepsin), the CD spectra analysis performed by means of the CDPro software [5] (data not shown) indicates that the $\alpha$-helix content increases even in 
all-alpha proteins (BSA, HSA, myoglobin). FTIR spectrum shows an enhancement of the component at $\sim 1657 \mathrm{~cm}^{-1}$ and a generalized reduction of the other component bands, thus indicating the predominance of the $\alpha$-helical structure.

In conclusion, the experimental results stress once more that hydration water is not just surrounding the protein, but, rather, it is interacting with. Water is responsible for both the features in the TSDC spectrum and the model of the water interacting with protein in different sorption sites may help to interpret the TSDC results by assuming the existence of energetically different families of water molecules. In particular, HT peak can be associated to water molecules external to the protein, in vicinity of the surface. The different shapes and positions exhibited by the peak for the six proteins in the native state may be accounted for both by different amounts and volumes of the cavities accessible to the solvent and by the hydrophobic/hydrophilic character of the cavity surfaces. By favoring the rearrangement of some portions of the structure and the formation of intramolecular hydrogen bonds in the polypeptide structure, by the water exclusion, TFE promotes the formation of the $\alpha$-helical secondary structure. The features of the TSDC spectra for TFE treated samples: (1) resemble those of dehydrated proteins and (2) are all very similar even for different proteins. This means that the conformational rearrangement achieved by the six proteins is the same, because each polypeptide makes available the same reduced population of binding sites to the solvent molecules. Since CD and FTIR measurements prove that the conformation attained is the $\alpha$-helix, the result emphasizes an enhanced stability of this structural motif in the dehydrated form of polypeptides, probably due to its local compactness.

\section{References}

[1] M.G. Bridelli and R. Capelletti, Hydration structure analysis of lysozyme amyloid fibrils by thermally stimulated depolarization currents (TSDC) technique, Spectroscopy 22 (2008), 165-176.

[2] M.G. Bridelli, R. Capelletti, F. Maraia, C. Mora and L. Pirola, Initial hydration steps in lipase studied by means of water sorption isotherms, FTIR spectroscopy and thermally stimulated depolarization currents, J. Phys. D: Appl. Phys. 35 (2002), 1039-1048.

[3] R. Capelletti and R. Fieschi, in: Electrets, M.M. Perlman, ed., The Electrochemical Society, Princeton, NY, 1972, pp. 1-10.

[4] R. Rajanand and P. Balaram, A model for the interaction of trifluoroethanol with peptides and proteins, Int. J. Peptide Protein Res. 48 (1996), 328-336.

[5] N. Sreerama and R.W. Woody, Computation and analysis of protein circular dichroism spectra, Meth. Enzymol. 383 (2004), 318-351. 


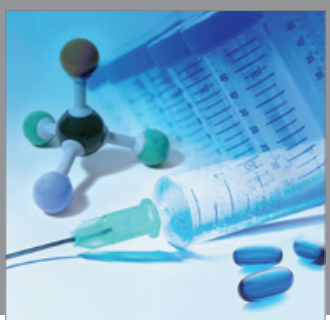

International Journal of

Medicinal Chemistry

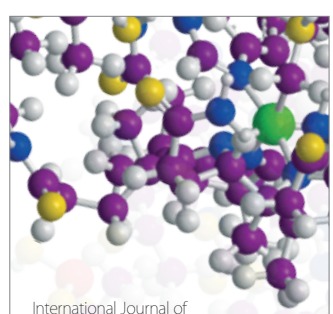

Carbohydrate Chemistry

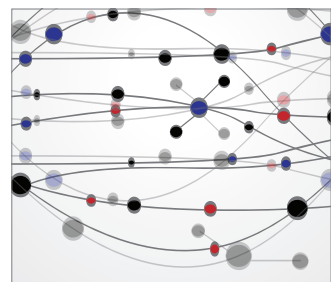

The Scientific World Journal
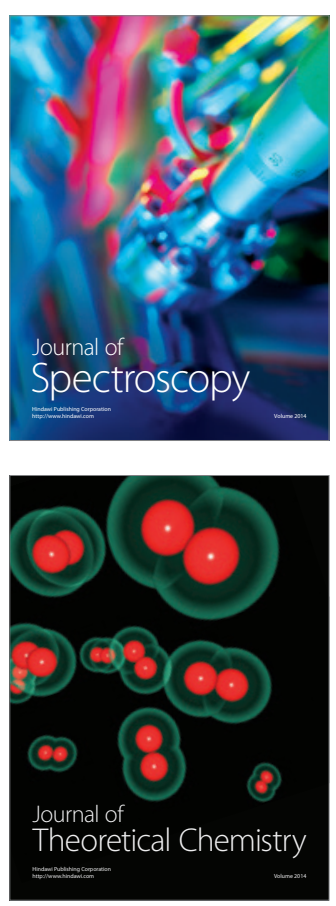
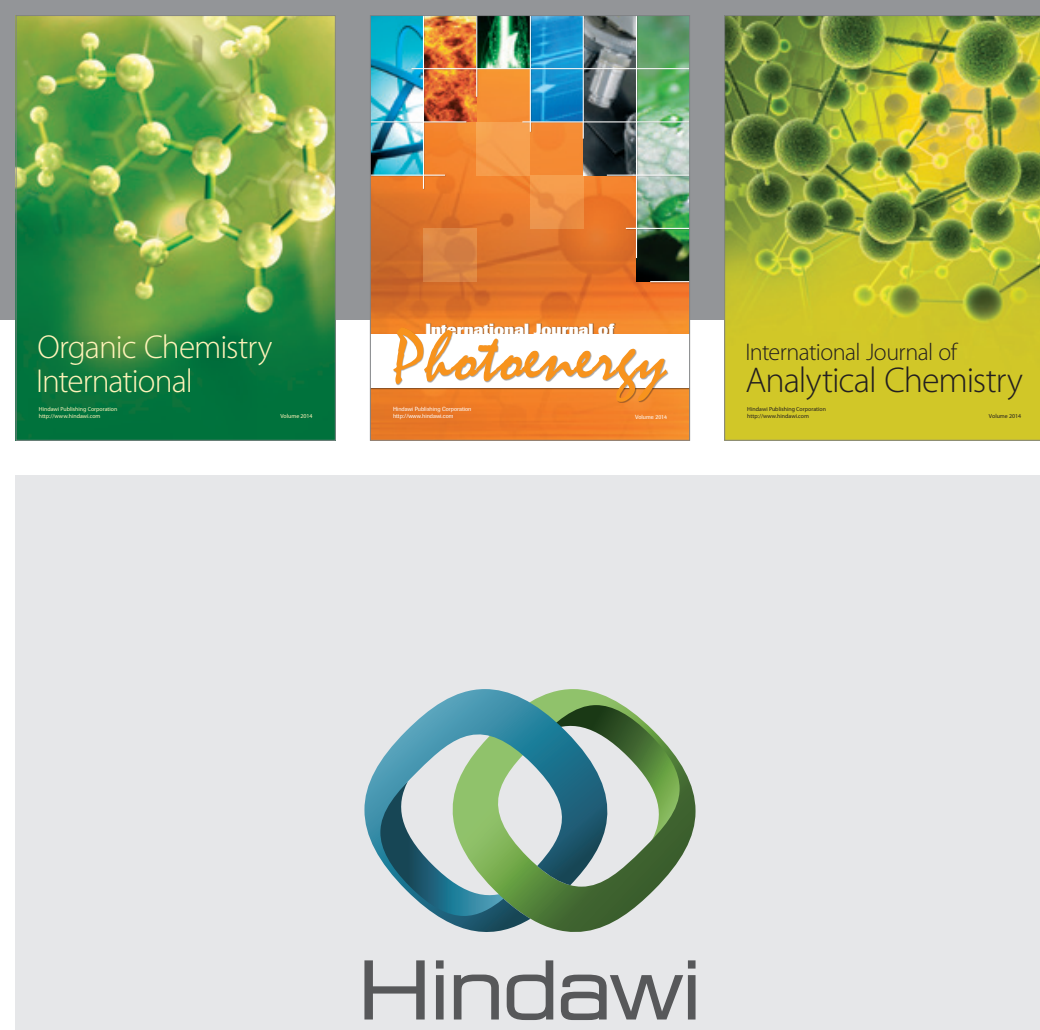

Submit your manuscripts at

http://www.hindawi.com
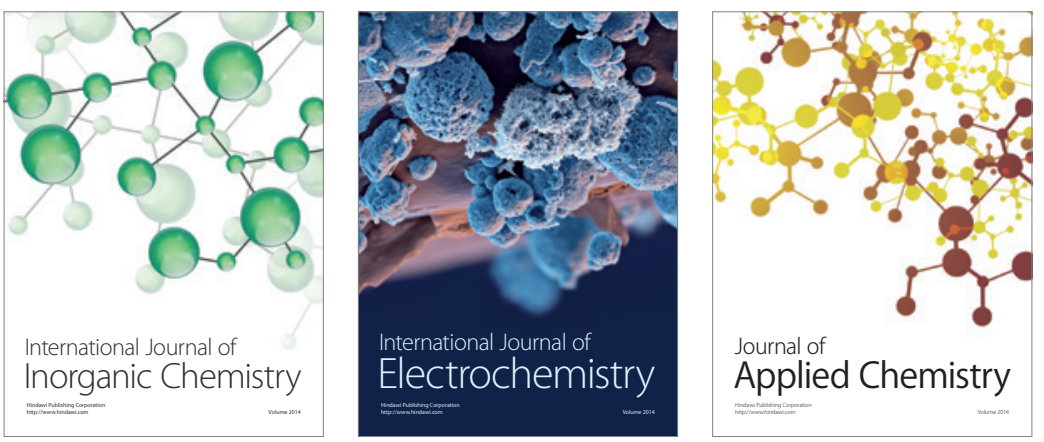

Journal of

Applied Chemistry
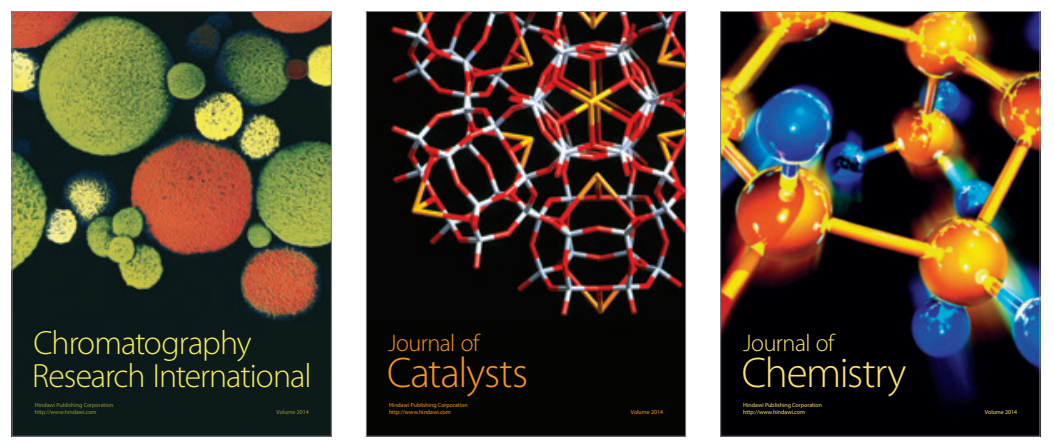
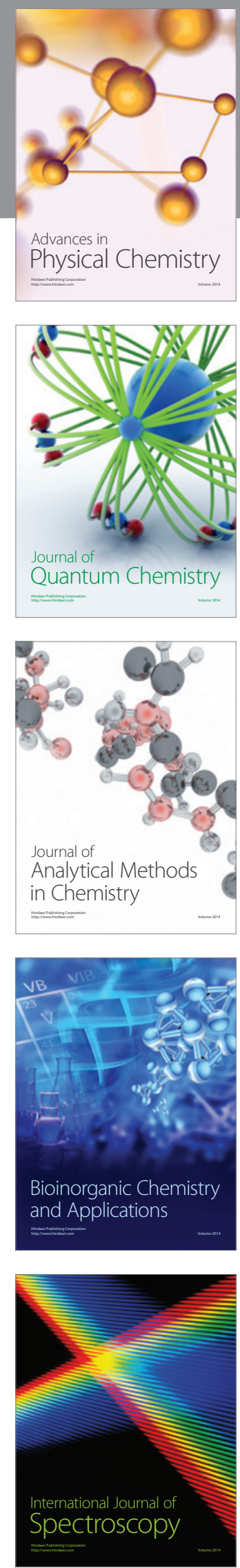